Eur. J. Clin. Chem. Clin. Biochem.

Vol. 30, 1992, pp. 503-511

(C) 1992 Walter de Gruyter \& Co. Berlin · New York

\title{
Towards an Understanding of the Mode of Action of Bacillus Calmette-Guérin-Therapy in Bladder Cancer Treatment, Especially with Regard to the Role of Fibronectin
}

\author{
By Esther van der Sloot ${ }^{1}$, S. Kuster ${ }^{2}, A$. Böhle $^{2}, J$. Braun $^{1}$ and W. G. Wood ${ }^{3}$ \\ 1 Klinik für Innere Medizin \\ ${ }^{2}$ Klinik für Urologie \\ Medizinische Universität zu Lübeck, Lübeck, Germany \\ ${ }^{3}$ Institut für Klinische Laboratoriumsdiagnostik \\ Klinikum der Hansestadt Stralsund, Stralsund, Germany
}

(Received January 21/May 22, 1992)

Summary: The interaction between Bacillus Calmette-Guérin (BCG) and the host was investigated after repeated intravesicular BCG-therapy for superficial bladder cancer. Studies were performed on

(a) the local reaction in the bladder,

(b) the systemic reaction, and

(c) short and long term interactions in both the bladder and the serum/plasma.

The analytes measured included anti-BCG IgA and IgG, fibronectin, lactoferrin, elastase- $\alpha_{1}$-proteinase inhibitor, myeloperoxidase and $\alpha_{2}$-proteinase inhibitor. All analytes, with the exception of $\alpha_{2}$-proteinase inhibitor, were measured in both serum/plasma and urine.

An additional group of 94 patients undergoing bronchoalveolar lavage was used for comparison with other diseases affecting mucous membranes. In vitro studies on human bladder in culture were also carried out to study the relationship between BCG, elastase and fibronectin.

The results revealed a normal defence reaction, in which $\operatorname{IgA}$ and $\operatorname{IgG}$ antibodies specific to BCG were produced by the host. Maximal concentrations of all analytes in urine were found about $4 \mathrm{~h}$ after BCG instillation.

Immunoglobulins, soluble fibronectin, and granulocyte markers all appeared in urine after instillation and all showed a similar time course. The in vitro study showed the synergistic effect of elastase and BCG in stimulating the host defence reaction. The relationship between BCG and fibronectin can be seen as fortuitous but not indicative of the efficacy of BCG-therapy in patients with superficial bladder cancer.

\section{Introduction}

Although post-operative intravesicular treatment of superficial bladder cancer with Bacillus CalmetteGuérin (BCG) has been carried out for some 15 years (1) the exact mode of action of this therapy is still not known. Many theories have been postulated, including the interaction between BCG and fibronectin
$(2-4)$, the necessity for the presence of a fibrin clot $(3,5)$, the saturation of BCG receptors with soluble monomeric fibronectin (6), and the mediation of $\mathrm{T}$ lymphocytes (7). Studies in these laboratories over the past four years have revealed further physiological factors which may contribute to the action of BCG in promoting an "anti-tumour" reaction in cases operated for superficial bladder cancer. 
The studies were carried out to investigate

(a) the nature of the immune response and its relationship to time, as well as to repeated BCG instillation,

(b) the relationship between BCG and fibronectin, and

(c) the role of leukocyte infiltration and leukocyte products - especially proteinases - in creating an optimal milieu for bladder wall permeation of BCG and the initiation of antigen-antibody reactions.

\section{Materials and Measurements \\ Materials}

Antibodies to BCG, fibronectin, human IgG and IgA, elastase, $\alpha_{1}$-proteinase inhibitor, $\alpha_{2}$-proteinase inhibitor, lactoferrin and myeloperoxidase were purchased either from DAKO (Hamburg, D), Serotec (Camon, Wiesbaden, D) or Atlantic Antibodies (Baxter, Munich, D). All were used as purified IgG fractions and were, according to the manufacturers, "monospecific" in nature. No further purification was carried out.

Labelling was carried out using either a biotin derivative (8) or the luminogen 9-[4-aminobutyl- $\mathrm{N}$ ethyl]-aminobenzo (f) phthalazine $1,4,(2 \mathrm{H}, 3 \mathrm{H})$ dione (ABEN (9)) as published in detail elsewhere. Assays were carried out using polystyrene balls (6.4 $\mathrm{mm}$ diameter) as solid phase (Spherotech Kugeln, Fulda, D).

Measurements were performed in a 250 sample semiautomatic luminometer with on-line data reduction (LB 952 16T, EG \& G Berthold, Wildbad, D).

\section{Methods}

The analyses were carried out using immunoluminometric assays (10). The analytes included:

antibodies to BCG (human class IgA and IgG),

antibodies to fibronectin (human class $\operatorname{IgA}$ and $\mathrm{IgG}$ ),
Tab. 1a. Immunoluminometric assay of urinary fibronectin

$10 \mu$ sample

$200 \mu$ l assay buffer

1 Anti fibronectin coated ball (rabbit-IgG fraction)

Incubate at ambient temperature $\left(18-25^{\circ} \mathrm{C}\right)$ for $60 \mathrm{~min}$ on horizontal rotator $\left(170 \mathrm{~min}^{-1}\right)$.

Wash with $2 \times 5 \mathrm{ml}$ demineralised water.

$200 \mu \mathrm{l}$ ABEN-labelled anti fibronectin (goat-IgG fraction)

Incubate and wash as above, transfer ball to measuring cuvette, add $300 \mu \mathrm{l}$ catalase and load luminometer.

Initiate light reaction with $\mathrm{NaOH} / \mathrm{H}_{2} \mathrm{O}_{2}$ and integrate signal over $2 \mathrm{~s}$.

Tab. 1b. Immunoluminometric assay to measure specific antibodies to BCG

$10 \mu$ sample (undiluted urine, serum IgA $1: 5 / 1: 10$, IgG $1: 10 / 1: 20)$

$200 \mu l$ assay buffer

1 ball with BCG covalently coupled antigen mixture

Incubate and wash as in table 1a above.

$200 \mu \mathrm{l}$ ABEN labelled anti serum IgA or IgG.

Incubate and wash as above, transfer ball to cuvette and proceed as in table 1a, integrating the light signal for $4 \mathrm{~s}$.

fibronectin, albumin, transferrin, elastase, $\alpha_{1}$-proteinase inhibitor, $\alpha_{2}$-proteinase inhibitor, lactoferrin and myeloperoxidase.

The solid phase was coated with antigens and antibodies as previously described $(8-10)$.

The assay schemes for antibody detection and antigen detection are shown in tables $1 \mathrm{a}$ and $1 \mathrm{~b}$ for urinary fibronectin and antiBCG-IgA/IgG. The assay schemes for the other analytes were similar.

Tab. 2. Ranges covered by assays, together with $95 \%$ confidence limits for healthy volunteers

\begin{tabular}{|c|c|c|c|}
\hline Analyte & Measuring range & $95 \%$ confidence limits & Material \\
\hline Elastase & $5-1900 \mu \mathrm{g} / 1$ & $\begin{array}{l}<210 \mu \mathrm{g} / 1 \\
\text { undetectable }\end{array}$ & $\begin{array}{l}\text { Plasma } \\
\text { Urine }\end{array}$ \\
\hline Myeloperoxidase & $5-1200 \mu \mathrm{g} / 1$ & $\begin{array}{l}<240 \mu \mathrm{g} / 1 \\
\text { undetectable }\end{array}$ & $\begin{array}{l}\text { Plasma } \\
\text { Urine }\end{array}$ \\
\hline Lactoferrin & $5-3000 \mu \mathrm{g} / 1$ & $\begin{array}{l}<250 \mu \mathrm{g}(1 \\
<20 \mu \mathrm{g} / \mathrm{mmol} \\
\text { creatinine }\end{array}$ & $\begin{array}{l}\text { Plasma } \\
\text { Urine }\end{array}$ \\
\hline Fibronectin & $10-2800 \mu \mathrm{g} / 1$ & $\begin{array}{l}\quad 250-600 \mathrm{mg} / \mathrm{l} \\
<\quad 30 \mu \mathrm{g} / \mathrm{mmol} \\
\text { creatinine }\end{array}$ & $\begin{array}{l}\text { Plasma } \\
\text { Urine }\end{array}$ \\
\hline Anti BCG IgA & $5-100 \mathrm{U} / 1^{*}$ & $\begin{array}{l}<110 \mathrm{U} / 1^{*} \\
\quad 6-18 \times 10^{6} \mathrm{U} / \mathrm{mol} \\
\text { creatinine }\end{array}$ & $\begin{array}{l}\text { Serum } \\
\text { Urine }\end{array}$ \\
\hline Anti BCG IgG & $5-100 \mathrm{U} / 1$ & $\begin{array}{l}<95 \mathrm{U} / 1 \\
\quad 8-40 \times 10^{6} \mathrm{U} / \mathrm{mol} \\
\text { creatinine }\end{array}$ & $\begin{array}{l}\text { Serum } \\
\text { Urine }\end{array}$ \\
\hline
\end{tabular}

* U - arbitrary units 
Table 2 shows the concentration ranges covered by each assay and the reference ranges in plasma and urine for healthy volunteers.

Standards for the BCG-antibody assays were prepared from antibody-positive urine. Standards for fibronectin were prepared from human soluble fibronectin (Calbiochem - Behring, Frankfurt a. M., D). Other standards were purchased or prepared as necessary $(8-10)$

\section{Experimental concepts and details}

a) The detection of specific antibodies to BCG in the urine and plasma of BCG-instilled patients - long and short term studies

Patients were monitored,

1. in a longitudinal study over 12 months in which blood and urine samples were taken before the BCG-instillation, and

2 . in a short-term study covering the $\mathbf{2 4}$ hours starting directly before the 6th instillation.

In the first case, 6 cycles of therapy were monitored for residual antibody activity in blood and urine. In the 24 hour study, blood and urine samples were collected directly before and at 5 additional times $(2,4,6,8$ and $24 \mathrm{~h})$ after the 6 th instillation. Although the 24 hour study was carried out for different instillation cycles, only the results from the final cycle are shown here. Total IgG and IgA were measured, as well as anti-BCG specific $\operatorname{IgG}$ and $\operatorname{IgA}$, in both serum and urine.

b) Detection of soluble fibronectin in urine and plasma in patients undergoing BCG-therapy

Fibronectin was measured using the same two time regimes described for BCG-antibodies in a) above. Both long and shortterm studies using plasma and urine samples were carried out. Detection of anti-fibronectin was always found to be negative.

c) Detection of the leukocyte markers, elastase- $\alpha_{1}$-proteinase inhibitor complexes, lactoferrin and myeloperoxidase in plasma and urine

The three markers for granulocyte infiltration and activity were assayed in urine samples using the same patients and the same two time regimes described in a) above.

d) Detection of serum-specific analytes in urine to confirm/exclude microhaematuria

$\alpha_{2}$-Proteinase inhibitor, transferrin and IgG were measured in serum and urine, in order to detect any lesions in the bladder or ureter which would lead to "false positive" values for fibronectin in urine. The ratio between these proteins in serum and in urine confirms the presence or absence of their passive transfer from the blood stream into the bladder. Only data from patients, in which haematuria had been excluded, were used in this study.

e) Control with patient groups with benign bladder disorders and with healthy volunteers

Controls were chosen from patients with urosepsis, cystitis and other benign bladder disorders, as well as healthy individuals who showed a normal urine sediment.

f) In vitro studies on the interaction of BCG with elastase and with fibronectin in human bladder culture

The production/interaction of BCG, fibronectin and elastase was studied in vitro, using sterile, freshly isolated human bladder pieces in culture over a 24 hour period. The presence and/ or production of elastase- $\alpha_{1}$-proteinase inhibitor complexes, fibronectin, myeloperoxidase and lactoferrin was studied. $\alpha_{2}$-Macroglobulin was chosen as a "serum specific analyte" to monitor the presence/release of blood-vessel contents into the medium.

Single pieces (ca. $5 \mathrm{mg}$ ) of healthy human bladder, obtained during cystectomy, were immediately transferred to and incubated in $5 \mathrm{ml} \mathrm{RMPI} 1640$ medium pH 7.4 at $37^{\circ} \mathrm{C}$ in the presence of $5 \% \mathrm{CO}_{2}$. RMPI 1640 medium alone served as medium control. Bladder in isotonic Ringer solution $\mathrm{pH} 7.4$ served as autolysis control. BCG (ca. $200 \times 10^{9} \mathrm{U} / \mathrm{l}$ ), elastase (final concentration $450 \mu \mathrm{g} / \mathrm{l}$ ) and BCG and elastase were included in three incubation chambers to investigate and simulate in vivo situations. Bladder in Hank's medium served as tissue control. After incubation, the pieces of bladder were embedded for histology and sections stained with haematoxylin-eosin and auromycin.

g) Comparison with other known diseases where granulocyte activity is detectable

Samples of bronchoalveolar lavage from patients with acute pulmonary disease (sepsis, inflammation) and non-inflammatory lung disorders were analysed for the three granulocyte markers (see c) above) as well as for soluble fibronectin.

The studies were carried out over a four year period, the most important results being summarised in this publication.

\section{Statistics}

Non-parametric statistics were used throughout. For the small numbers of patients in each group the $\chi^{2}$ test was replaced by the exact Fisher test, which is designed for studies with $\mathrm{n}<60$ data. The Mann-Whitney U-Test was used for independent variables, the Wilcoxon signed rank test for paired data, and the Spearman rank correlation for the investigation of concordance between two related groups. The median was used as the central tendency, together with the relevant percentiles as measures of dispersion.

Results

1) The course of anti-BCG antibody production in the bladder during $B C G$ therapy and the appearance of specific anti $B C G$ immunoglobulins in blood

During the course of six instillations of BCG at 4 week intervals, IgA and IgG antibodies to BCG measured prior to instillation were as shown in figure 1. The time course of production of antibodies was followed for $24 \mathrm{~h}$ after the 6th instillation by measuring the $\operatorname{IgA}$ and $\operatorname{IgG}$ contents in the urine voided at the time intervals shown. Both total immunoglobulins and BCG-specific immunoglobulin antibodies were measured. The BCG-specific antibodies are shown in figure $2 a$ (urine) and figure $2 b$ (serum). The total IgA and IgG in serum did not change during therapy or after instillation. The IgA and IgG in urine was almost exclusively anti-BCG-specific. 


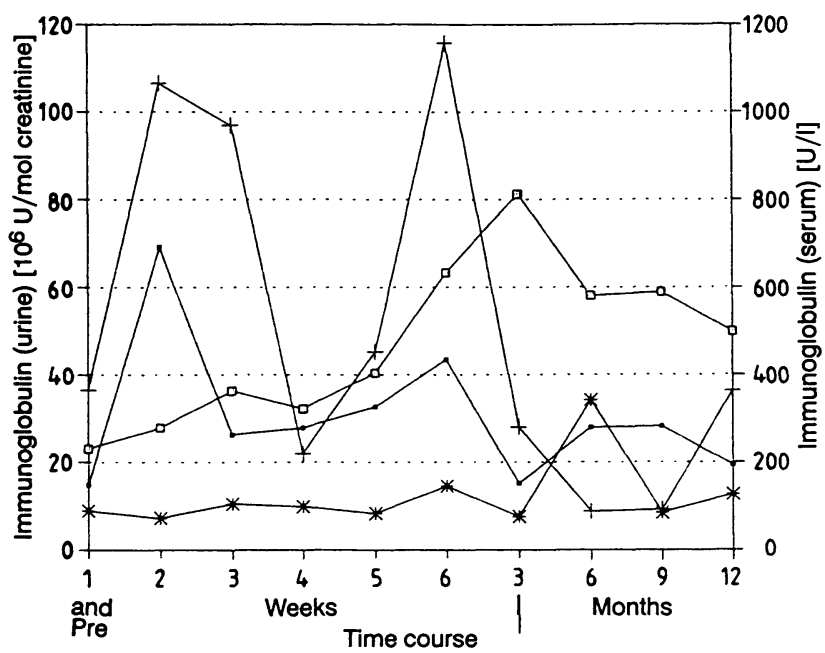

Fig. 1. Anti BCG $\operatorname{IgA}$ and $\operatorname{IgG}$ in serum and urine during instillation therapy.

Time course of antibody titres to BCG in serum and urine. The measurements were made prior to each instillation $\left(1^{\text {st }}-6^{\text {th }}\right.$ week) and at the control check-ups 3 , 6,9 and 12 months after the start of therapy.

- IgA, urine

* IgA, serum

$+\mathrm{IgG}$, urine

口 IgG, serum

Only IgA and IgG specifically directed against a BCGhomogenate were measured. The median values from 10 patients are shown. Due to large individual differences, no dispersion parameters are shown.

2) Concentrations of fibronectin in the urine of patients under the same treatment as in (1) above

The immunoluminometric assay was capable of measuring less than $15 \mu \mathrm{g} / \mathrm{l}$ and was therefore ideal for urinary estimations. Figure 3 shows the median concentrations of urine fibronectin in 12 patients after the 6 th instillation (over $24 \mathrm{~h}$ ). The concentrations of soluble fibronectin found in urine before each instillation were constant $(25 \pm 4 \mathrm{mg} / \mathrm{mol}$ creatinine $)$. There was no significant difference between the values before each instillation ( $p>0.7)$. As stated above, antibodies to soluble fibronectin were never found in urine.

3) The production of the leukocyte components, elastase, myeloperoxidase and lactoferrin during BCG instillation, and their relationship to fibronectin and anti-BCG

The invasion of the bladder by leukocytes (mainly B lymphocytes and polymorphonuclear neutrophils), after instillation, led to the appearance of known markers for these cells both longitudinally and over 24 hours.
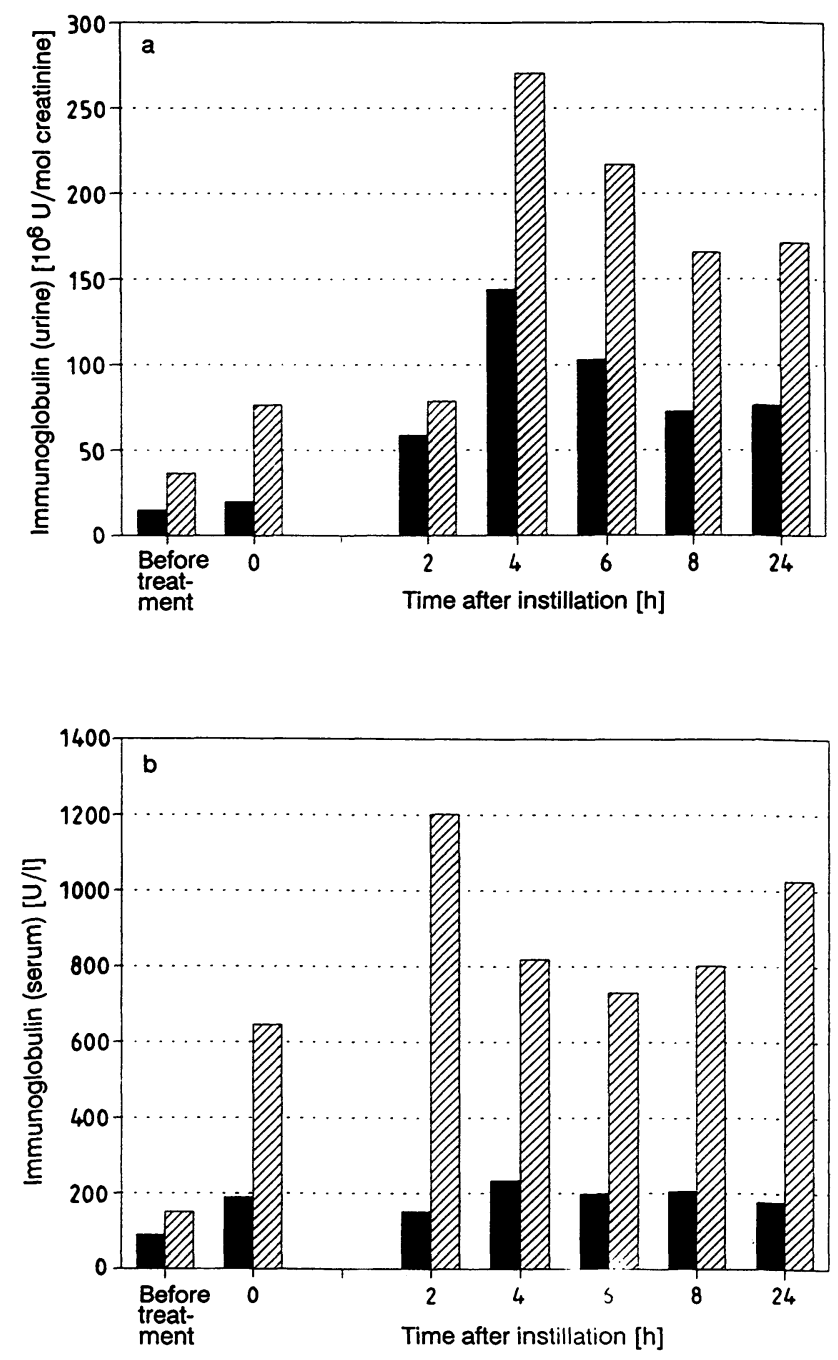

Fig. 2. Anti BCG IgA ( $\square$ ) and IgG (ש) in urine (a) and serum (b) after the 6th instillation.

The acute response to BCG instillation in the bladder is shown. Anti-BCG specific IgA and IgG were measured before and up to 24 hours after the 6th BCG instillation.

Before treatment - values before commencement of treatment (pre 1st instillation),

0 - value directly before the 6 th instillation,

Only IgA and IgG specifically directed against a BCGhomogenate were measured. The median values from 10 patients are shown. Due to large individual differences, no dispersion parameters are shown.

The results for the $24 \mathrm{~h}$ study are shown in figure 3 and are median values for 12 patients undergoing the same treatment as in (1) above. Direct leakage of serum is excluded, because transferrin, IgG and $\alpha_{2}-$ proteinase inhibitor concentrations in urine do not change significantly during the 24 hour period studied (results not shown). The levels before instillation were not significantly different from those found in urine from hospitalised patients without known bladder disease. 


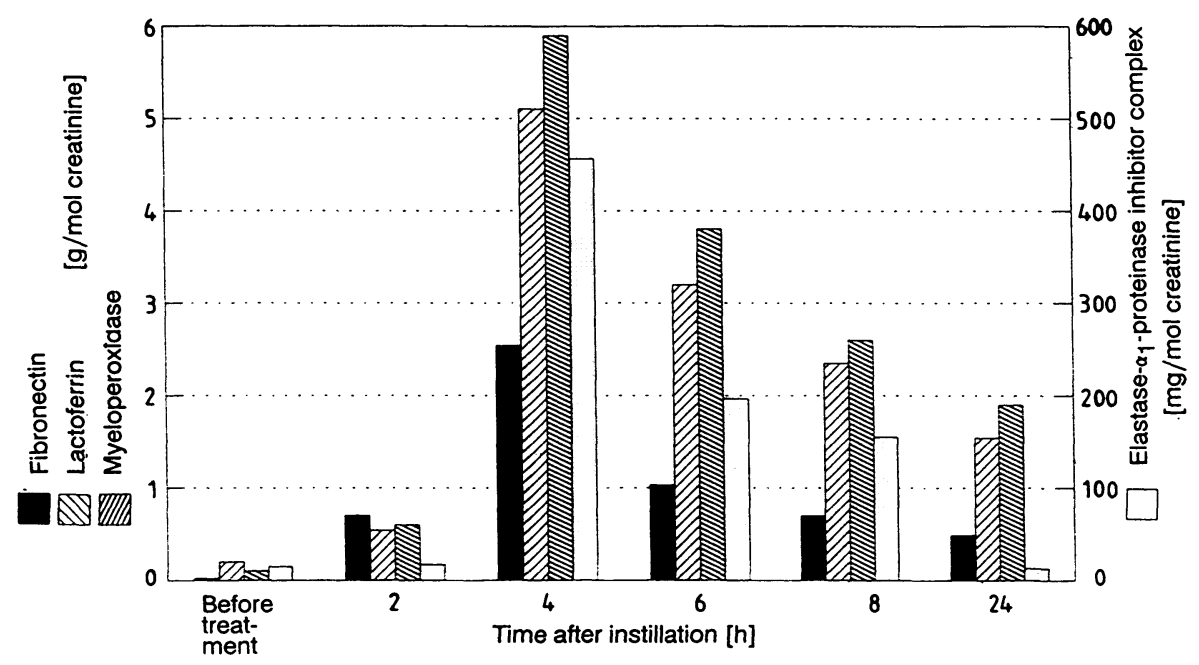

Fig. 3. Urinary concentrations of fibronectin, lactoferrin, myeloperoxidase and elastase- $\alpha_{1}$-proteinase inhibitor complex after the 6th instillation.

The release/appearance of fibronectin, lactoferrin, myeloperoxidase and elastase- $\alpha_{1}$-proteinase inhibitor complex in urine directly before and up to $24 \mathrm{~h}$ after the 6th BCG instillation is shown. Note that only elastase complexed with $\alpha_{1}$-proteinase inhibitor was measured; free elastase was not determined. The results are the median values from 12 patients.

\section{4) Interaction between $\mathrm{BCG}$ and elastase in vitro}

Figures $4 a-4 e$ show the concentrations in the different incubation chambers for elastase- $\alpha_{1}$-proteinase inhibitor complexes, lactoferrin, myeloperoxidase, fibronectin and $\alpha_{2}$-proteinase inhibitor respectively. As the medium contained $530 \mu \mathrm{g} / 1$ fibronectin (Fig. 4c) this concentration was subtracted from the actual concentrations in each well so that the net production of fibronectin is shown in figure 4c.
As was to be expected, the medium itself showed no changes in concentration and little or no native analyte (with the exception of fibronectin).

The results in the well containing bladder and Ringer solution showed that autolysis took place, the most marked results being those for myeloperoxidase, $\alpha_{2}$ proteinase inhibitor and fibronectin. The histology confirmed the autolysis.

The well containing the RPMI 1640 medium and bladder showed relatively little autolysis, only mye-

Tab. 3. Relationship between elastase, lactoferrin, myeloperoxidase and fibronectin in bronchoalveolar lavage in 94 patients with pulmonary infection

a) Concentrations in $\mu \mathrm{g} / \mathrm{l}$

\begin{tabular}{|c|c|c|c|c|}
\hline Percentiles & Elastase & Lactoferrin & Myeloperoxidase & Fibronectin \\
\hline 2.5 & $<5$ & $<5$ & $<5$ & 20 \\
\hline 16 & 5.2 & 25 & 7.0 & 100 \\
\hline Median & 26 & 87 & 28 & 260 \\
\hline 84 & 121 & 577 & 420 & 640 \\
\hline 97.5 & 2700 & 5790 & 13000 & 9700 \\
\hline Range & $<5-3160$ & $<5-10130$ & $<5-46800$ & $20-10000$ \\
\hline
\end{tabular}

b) Correlation coefficients (r)

\begin{tabular}{llll}
\hline Elastase & vs. & Lactoferrin & $=0.519$ \\
Elastase & vs. & Myeloperoxidase & $=0.795$ \\
Elastase & vs. & Fibronectin & $=0.592$ \\
Lactoferrin & vs. & Myeloperoxidase & $=0.721$ \\
Lactoferrin & vs. & Fibronectin & $=0.520$ \\
Myeloperoxidase & vs. & Fibronectin & $=0.622$ \\
\hline
\end{tabular}

$\mathrm{p}<0.001$ in all cases 

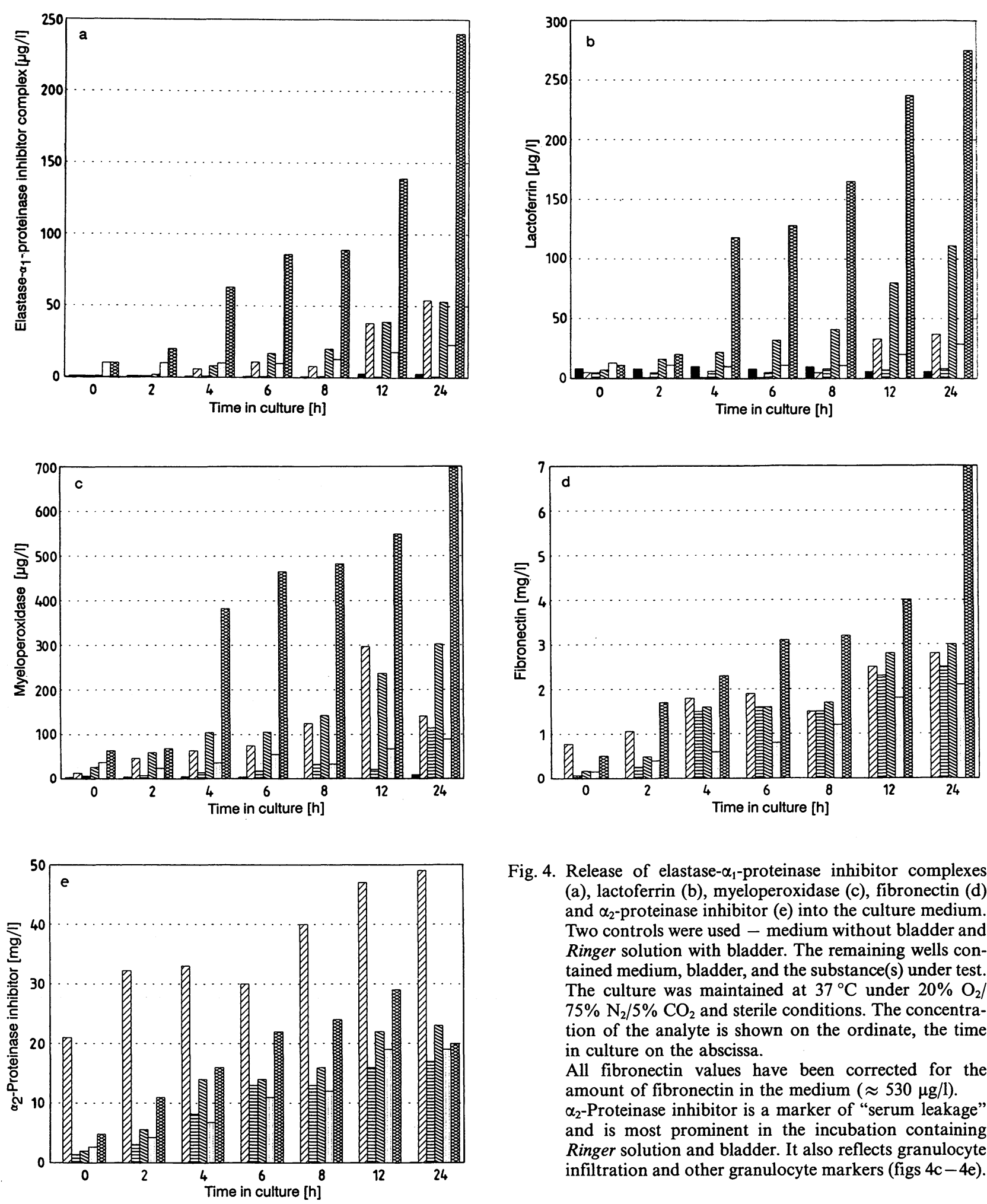

Fig. 4. Release of elastase- $\alpha_{1}$-proteinase inhibitor complexes (a), lactoferrin (b), myeloperoxidase (c), fibronectin (d) and $\alpha_{2}$-proteinase inhibitor (e) into the culture medium. Two controls were used - medium without bladder and Ringer solution with bladder. The remaining wells contained medium, bladder, and the substance(s) under test. The culture was maintained at $37^{\circ} \mathrm{C}$ under $20 \% \mathrm{O}_{2} /$ $75 \% \mathrm{~N}_{2} / 5 \% \mathrm{CO}_{2}$ and sterile conditions. The concentration of the analyte is shown on the ordinate, the time in culture on the abscissa.

All fibronectin values have been corrected for the amount of fibronectin in the medium $(\approx 530 \mu \mathrm{g} / \mathrm{l})$. $\alpha_{2}$-Proteinase inhibitor is a marker of "serum leakage" and is most prominent in the incubation containing Ringer solution and bladder. It also reflects granulocyte infiltration and other granulocyte markers (figs $4 c-4 e$ ).

medium

Ringer/bladder medium/bladder
BCG/bladder loperoxidase, $\alpha_{2}$-proteinase inhibitor and fibronectin being released into the medium.

The well containing the BCG showed continuous release of all 5 analytes, showing that in vitro BCG leads to stimulation of the granulocytes present in the biopsy material.

The well containing the polymorphonuclear granulocyte elastase showed less response than that with 
BCG. The combined effect of BCG and elastase gave rise to the largest responses in four analytes. Bacterial contamination of the culture medium was negative after $24 \mathrm{~h}$ so that non-specific effects can be excluded.

All concentrations are the mean of duplicate determinations, as this experiment was carried out only once.

5) The measurement of elastase, myeloperoxidase, lactoferrin and fibronectin in bronchoalveolar lavage of patients with pulmonary infections

This was performed to see how the above mentioned analytes acted in a natural infection process occurring at a mucosal barrier. The 94 patients involved in the study were undergoing routine lavage inspection. Table 3 shows the ranges and median concentrations of each analyte in $\mu \mathrm{g} / \mathrm{l}$ as well as the correlation between each component using the Spearman rank correlation coefficient.

Again, direct leakage from serum can be excluded, because the serum: lavage ratio of each analyte is not constant.

\section{Discussion}

The discussion compares known facts (which are not necessarily directly related to the bladder and BCG therapy) with the results of the investigation.

BCG therapy only appears to work effectively with live bacteria (11), so it can assumed that a vital component is necessary for successful therapy. It is known that BCG produces and releases a specific fibronectin-binding protein $\left(M_{\mathrm{r}} 32000\right)$ which is extremely antigenic in nature (12) as well as fibronectinbinding proteins $(13,14)$ of relative molecular mass $M_{\mathrm{r}} 85000$.

It is known also that myeloperoxidase, one of the neutrophil enzymes, can irreversibly oxidize fibronectin and make it more susceptible for degradation by elastase, another neutrophil enzyme (15).

In bacterial infection due to Gram-negative organisms, plasma fibronectin decreases at an early stage (16). Decreases have also been noted in pulmonary hypertension after oesophageal cancer operations (17). In both cases plasma elastase rose significantly showing an inverse relationship between elastase and fibronection. Treatments which suppress elastase release, for example administration of dexamethasone, result in elevated fibronectin values in bronchial lavage (18). Recent results using different cytokines have con- firmed that the degradation of fibronectin in an extracellular matrix is largely due to polymorphonuclear leukocyte elastase (19).

The effects of elastase on the degradation of basement membranes may assist the extravasation of neutrophils in the process of inflammation (20).

Other bacteria such as Pseudomonas sp. and Vibrio sp. produce structurally related elastases which are able to degrade fibronectin, lactoferrin and ovomucin (21).

Several deductions and postulates can be made from these observations and the results of the present study.

The removal of fibronectin from the basal membrane increases permeability to invading organisms possessing elastase-like exoproteinases, as well as to granulocytes and polymorphonuclear leukocytes which represent the natural cellular defence system of the host.

The oxidation of fibronectin by myeloperoxidase increases the susceptibility to degradation by elastase.

The mycobacteria have at least one specific fibronectin binding protein which shows a long phylogenetic association between host and invader. The attachment of BCG to fibronectin has also been documented by Ratliff and co-workers $(3,4)$ as well as other groups $(2,6)$. This would provide the BCG with an anchor so that its exoproteases can act to permeate the epithelium, thus allowing invasion of the organism.

In the later phase of BCG "attack", as in the later stages of the therapy, two reactions take place simultaneously: polymorphonuclear leukocytes of the host defence system release elastase and myeloperoxidase, while the mycobacteria release, amongst other things, their fibronectin binding protein.

The following may be postulated:

The host defence cells release BCG-specific antibodies of both $\operatorname{IgA}$ and $\operatorname{IgG}$ classes. The $\operatorname{IgA}$ antibodies attach themselves to BCG and lead to lysis of the mycobacteria.

The phagocytes release elastase, which dissolves not only bacterial proteins but also host proteins, especially membrane-incorporated fibronectin, which then competes with insoluble fibronectin for the BCG.

The good correlation between both bladder polymorphonuclear granulocyte elastase and lavage elastase and fibronectin concentrations tends to support the fact that the polymorphonuclear granulocyte elastase is probably responsible for the soluble fibronectin (19). 
The production of antibodies to surface components of BCG, as well as to internal proteins (12) leads to the following proposal.

The BCG which permeate or infiltrate the bladder wall are attacked by the natural host reaction, i. e. by anti-BCG immunoglobulins if the BCG is free in the bladder, or by phagocytes and T-cells if surrounded by tissue (granulomata).

The results from the isolated bladder experiments support the theory that although elastase alone leads to the production of fibronectin, the presence of BCG is necessary to initiate the activation of the leukocytes. This is reflected by the fact that the production of the analytes in the presence of elastase and BCG is greater than the sum of the products when elastase and BCG are added separately.

Another interesting feature of the in vitro study on fresh isolated bladder is that the leukocytes reacted exactly as in vivo, despite the fact that no perfusion was performed. The release of contents or opening of the blood vessels to the medium was well documented by the behaviour of $\alpha_{2}$-proteinase inhibitor, both in medium and Ringer solution.

The rapid appearance of this analyte reflected the autolysis process occurring in this control and explained the rapid appearance of some granulocyte products in the Ringer solution. The rate of their appearance also reflected the osmotic stability of the granula in which they are stored.

The results are in accordance with the isolated observations listed above and appear to explain the relationship between BCG and fibronectin in BCG instillation in the bladder.

Two types of response are seen in BCG instillation therapy:

a) The normal antigen-antibody response reflected in the production of IgA and IgG antibodies is specifically directed against BCG. This immune reaction type is typical for bacterial invasion via the mucosal membranes.

b) An indirect (Type IV) reaction is seen in the formation of granulomata in the bladder epithelium. This reaction is typical for mycobacteria.

The normal immunogenic reaction occurs as a response to the repeated instillation of large numbers of BCG, and it follows the typical course seen in immunisation, i. e. primary response (IgA production at mucous membrane barriers) followed by the longterm response (IgG production) (see fig. 1; bi-phasic response of antibody production). The type IV reaction may explain the anti-tumour effect of BCG therapy, since phagocytes and T-cells are both involved. If the granuloma also includes tumour cells, it is possible that the phagocytosis releases antigens or "tumour-cell epitopes", which are then recognised as "foreign" and thus give rise to a second, independent "immunisation process", leading to production of antibodies to tumour cell components.

Although this can be seen as being mediated by the BCG therapy, it is completely independent of it as far as the actual immune response is concerned.

The lymphocytes then not only recognise BCG but also anti-cell surface antibodies which leads to the lysis and destruction of the tumour cells. This is also seen in adjuvant tumour therapy, where the host receives his own tumour cells in an "appetising" form for phagocytes.

In such a case the role of fibronectin in BCG therapy can be seen as minimal and the relationship between BCG and fibronectin as phylogenetically historical and almost fortuitous in nature.

The bacterium binds the fibronectin to invade the host; at the same time the host sets in motion a "fibronectin solublisation process" to prevent the bacterium from being able to adhere to the epithelial cells, its specific binding protein being saturated with soluble fibronectin.

This would not only explain the results seen here, but would also explain the differing virulence of mycobacteria which appears to depend on the amount of fibronectin binding protein that they produce.

It also explains why dead BCG have little or no therapeutic effect as they are unable to permeate or attach to the epithelium and set the immune reaction in motion.

\section{Conclusions}

To conclude, it appears that to some extent the assumed obligatory association between BCG and fibronectin is mistaken, as far as explaining the mode of action of BCG instillation therapy is concerned. 


\section{References}

1. Morales, A., Eldinger, D. \& Bruce, A. W. (1976) Intracavitary bacillus Calmette-Guérin in the treatment of superficial bladder cancer. J. Urol. 116, 180-183.

2. Kavoussi, L. R., Brown, E. J. \& Ritchie, J. R. (1990) Fibronectin-mediated Calmette-Guérin bacillus attachment to murine bladder mucosa. J. Clin. Invest. 85, 62-67.

3. Ratliff, T. L., Palmer, J. O. \& Mc Garr, J. A. (1987) Intravesical bacillus Calmette-Guérin therapy for murine bladder tumors: initiation of the response by fibronectinmediated attachment of bacillus Calmette-Guérin. Cancer Res. 47, 1762-1766.

4. Ratliff, T. L., Mc Garr, J. A. \& Abou-Zeid, C. (1988) Attachment of Mycobacterium bovis to F-coated surfaces. J. Gen. Microbiol. 134, 1307-1313.

5. Grinnell, F., Billingham, R. E. \& Burgess, L. (1981) Distribution of fibronectin during wound-healing in vivo. $\mathrm{J}$. Invest. Dermatol. 76, 181-189.

6. Aslanzadeh, J., Brown, E. J. \& Quillin, S. P. (1989) Characterisation of soluble fibronectin binding to bacillus Calmette-Guérin. J. Gen. Microbiol. 135, 2735-2741.

7. Ratliff, T. L., Gillen, D. P. \& Catalona, W. F. (1987) Requirement of a thymus dependent immune response for BCG-mediated antitumor activity. J. Urol. 137, 155-158.

8. Wood, W. G. \& Missler, U. (1990) Setting up and optimising solid-phase luminescence immunoassays using 1,2-diacyl aryl hydrazides as labels. In: Luminescence immunoassay and molecular applications (Van Dyke, K. \& Van Dyke, R., eds) CRC Press, Boca Raton. pp. 141-156.

9. Wood, W. G., Fricke, H. \& Strasburger, C. J. (1988) Solid phase luminescence immunoassays using kinase and aryl hydrazide labels. In: Nonisotopic Immunoassay (Ngo, T. T., ed.) Plenum Press, New York, London. pp. 257-270.

10. Braun, J., Schultek, T., Tegtmeier, K. F., Florenz, A., Rohde, C. \& Wood, W. G. (1986) Luminometric assays of seven acute-phase proteins in minimal volumes of serum, plasma and bronchioalveolar lavage. Clin. Chem. 32, $743-$ 747.

11. Bartless, G. L., Kreider, J. W., Purnell, D. M. \& Katsilas, D. C. (1980) Augmentation of immunity to live 10 hepatoma by BCG: Comparison of different BCG preparations. Cancer (Phila) 3, 488-496.
12. Huygen, K., Palfliet, K., Jurion, F., Lenior, C. \& van Vooren, J. P. (1990) Antibody repertoire against culture filtrate antigens in wild house mice infected with Mycobacterium bovis BCG. Clin. Exp. Immunol. 82, 369-372.

13. Wiker, H. G., Harboe, M., Nagai, S. \& Bennedsen, J. (1990) Quantitative and qualitative studies on the major extracellular antigen of mycobacterium tuberculosis H37Rv and Mycobacterium bovis BCG. Am. Rev. Respir. Dis. 141, $830-838$.

14. Wiker, H. G., Sletten, K., Nagai, S. \& Harboe, M. (1990) Evidence for three separate genes encoding the proteins of the mycobacterial antigen 85 complex. Infekt. Immunol. $58,272-274$

15. Vissers, M. C. \& Winterbourn, C. C. (1991) Oxidative damage to fibronectin - I. The effects of the neutrophil myeloperoxidase system and $\mathrm{HOCl}$. Arch. Biochem. Biophys. 285, 53-59.

16. Kawai, S. (1991) Sepsis and ARDS. Nippon Kyobu Shikken Gakkai Zasshi 29, 153-158.

17. Sato, N., Muramaki, K., Ishida, K., Ikeda, K. \& Saito, K. (1991) Pulmonary hypertension and polymorphonuclear leucocyte elastase after esophagal cancer operations. Ann. Thorac. Surg. 54, 754-758.

18. Yoder, M. C., Jr., Chua, R. \& Tepper, R. (1991) Effect of dexamethasone on pulmonary inflammation and pulmonary function of ventilator dependent infants with bronchopulmonary dysplasia. Am. Rev. Respir. Dis. 145, 10441048.

19. Chamba, A., Afford, S. C., Stockley, R. A. \& Burnett, D. (1991) Extracellular proteolysis of fibronectin by neutrophils: Characterisation and the effects of recombinant cytokines. Am. J. Respir. Cell. Mol. Biol. 4, 330-337.

20. Watanabe, H., Hatton, S., Katsuda, S., Nakanishi, I. \& Nagai, Y. (1990) Human neutrophil elastase: degradation of basement membrane components and immunolocalisation in the tissue. J. Biochem. (Tokyo) 108, 753-759.

21. Hase, C. C. \& Finkelstein, R. A. (1990) Comparison of the Vibrio cholerae hemagglutinin/protease and the Pseudomonas aeruginosa elastase. Infect Immunol. 58, 40114015.

Prof. Dr. W. G. Wood

Institut für Klinische Laboratoriumsdiagnostik Klinikum der Hansestadt Stralsund

Krankenhaus am Sund

Große Parower Straße 47-53

O-2300 Stralsund

Bundesrepublik Deutschland 
